\title{
Method and software to perform Pitch Drop
}

\author{
Luiz Eduardo Guarino de Vasconcelos ${ }^{1,3}$, Nelson Paiva Oliveira Leite2, André Yoshimi Kusumoto², \\ Leandro Roberto ${ }^{5}$, Cristina Moniz Araujo Lopes ${ }^{1,4}$ \\ 1 Instituto Tecnológico de Aeronáutica (ITA), Brazil \\ 2 Instituto de Pesquisas e Ensaios em Voo (IPEV), Brazil \\ ${ }_{3}^{3}$ Instituto Nacional de Pesquisas Espaciais (INPE), Brazil \\ ${ }^{4}$ Instituto de Aeronáutica e Espaço (IAE), Brazil \\ ${ }^{5}$ Instituto de Estudos Avançados (IEAV), Brazil \\ du.guarino@gmail.com, epd@ipev.cta.br, andrekusumoto@gmail.com, leandrolr@fab.mil.br, \\ cmoniz77@gmail.com
}

\begin{abstract}
:
Store separation flight tests are considered high risk. Something that precedes the in-flight tests are the pitch drop tests. Pitch drop are fundamental because they allow the static evaluation that the separation meets a minimum degree of safety. A process that has added value to the experimental trials is the use of high data rate acquisition cameras (i.e. greater than $200 \mathrm{fps}$ ) and the use of three-dimensional software. In order to reconstruct the 3D separation path with $6 \mathrm{DoF}$ from images, 2 or more cameras are required. When using cameras in any activity that requires accuracy in the results, it is necessary to calibrate the optical system used in the tests. In Brazil, the IPEV carried out these tests for years, and the determination of the trajectory of store separation was carried out with a commercial software. In addition, the process was inefficient and costly as it required many weeks of work for the analysis of results and execution of flight tests. To eliminate the use of the commercial tool and to have technical mastery over the entire test, a solution was developed that (1) the construction of a calibration field with depth so that camera calibrations can be performed using a single frame; (2) a method for carrying out pitch drop; (3) and an application that uses computational vision to process the data and generate the results in 6DoF. The benefits of such a solution include better use of resources, minimization of workload, and reduced costs and time. In this paper, the calibration field, the method created for pitch drop, the steps for the development of the computational application and the experiments performed to validate the application are demonstrated.
\end{abstract}

Key words: image processing; store separation; real time; 3D analysis; camera calibration.

\section{Introduction}

The store separation of aircraft is an old story however the accuracy of the store trajectory during the separation of the aircraft is not so old [1]. Before the 1960s, there were practically no widely used or generally accepted methods for pre-flight prediction of store separation trajectories other than wind tunnel testing techniques. With the advent of modern attack aircraft high-speed, the need arose to carry more and more stores and release them at everincreasing speeds.

In a military aircraft, each external store (e.g. ammunition, external fuel tank, capsules, bombs, missiles, among others) must be released safely and the launch must be wellplanned [2]. To predict the store trajectories, simulation models with six DOF (degrees of freedom) are usually used that describe the movement of the aircraft and the store in relation to one another, as well as the inertial system.
There are two ways of determining flight trajectory data. One is photogrammetry and the other telemetry [3]. Both methods have advantages and disadvantages.

Pitch drop testing is a fundamental step in the store separation testing campaign [4]. These tests are done to establish the aircraft / store configurations required for flight tests. At the IPEV (Flight Testing and Research Institute), pitch drop testing were also essential for the development of a method and software that allows the analysis of store separation. At this time, this analysis is still done post processing. This is of fundamental importance for something that we have been aiming for 5 years, which is the near real-time analysis in the pitch drop testing and store separation flight tests. In this paper, we review some concepts about store separation, store separation analysis, photogrammetry and camera calibration. In addition, we show the use of a photogrammetric 
solution developed and used in a real pitch drop testing carried out at IPEV. We also show the implemented method and the pitch drop testing performed.

\section{Store Separation Analysis}

The store separation analysis is defined as the determination of position and attitude history of a store after it is deliberately detached or ejected from the aircraft while the store is still under nonuniform aerodynamic interference near the aircraft [2].

The store separation analysis is necessary to redesign the operational limit of store, called envelope [5], in order to analyze the store separation characteristics for various flight conditions.

It is important to note that the main purpose of the store separation test is to collect sufficient data to ensure acceptable separation in terms of safety [6].

\section{Photogrammetry}

Photogrammetry is the science of making accurate measurements from photographs [7]. This technique allows to obtain quantitative data of the cameras mounted in the aircraft. Quantitative data are essential to validate the models used in the store separation tests so that they could be used to improve the accuracy of store separation predictions. Usually, highspeed video cameras are used in these tests.

The use of photogrammetry in store separation tests offers significant and unique technical and managerial challenges. When designing the algorithms several factors must be considered, such as camera angle, camera movement, image quality, focal length, lens distortions and environmental conditions. Most of the time, the vapors appear on the images that may decrease the visibility or even obscure the charge. A camera may get engine fluid in its lens or the camera may malfunction and stop working completely.

In addition, store separation tests occur in environments hostile to accurate measurements. The light condition is a major challenge in the use of high-speed cameras [8].

Without photogrammetry, the in-flight test is of a qualitative nature and consists of several flights with store separation approaching the edge of the separation envelope in small incremental steps. This is extremely dangerous.

\section{Pitch Drop Testing}

In order to perform this test, the IPEV developed a method that is divided into the following steps: planning, preparation, geometry determination, camera calibration, test point realization, 2D analysis and 3D analysis with 6DOF..

\section{Planning}

As previously stated, a photogrammetric solution to store separation offers significant and unique technical and managerial challenges. At this stage, the planning of the entire test campaign is carried out and the following activities and information are defined: the test period; the teams that will participate in the campaign. Typically, teams from the imaging sector, aircraft maintenance sector, technical support sector, instrumentation and calibration sector, surveying sector; equipment to be used (aircraft, cameras, total station, luxmeter, trigger, targets, tires (Figure 1), power sources, computers, fork-lift, among others).

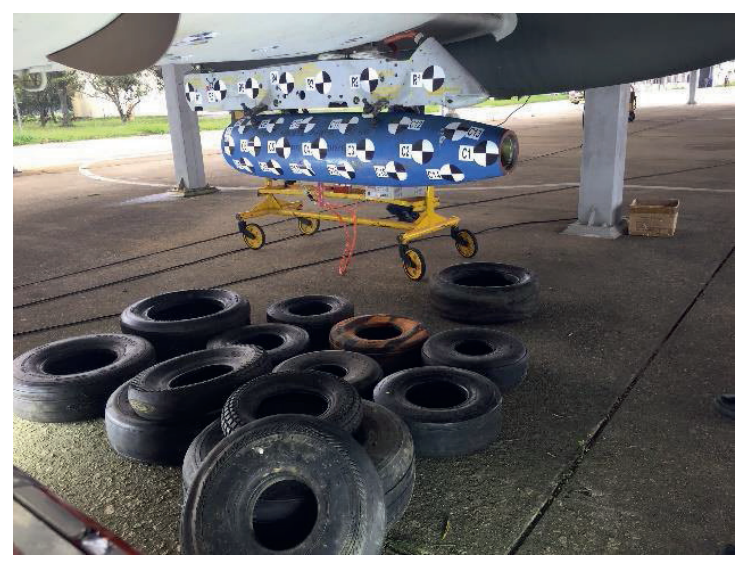

Fig. 1. Store coupled on the pylon and tires on the ground to cushion the fall.

The target is a circular or square 4 or 6 inch sticker with a bow tie feature (Figure 2). More information is placed close to each target to facilitate identification of the target (i.e. a letter and a number) (Figure 2). This identification facilitates the possible post-processing of the data.

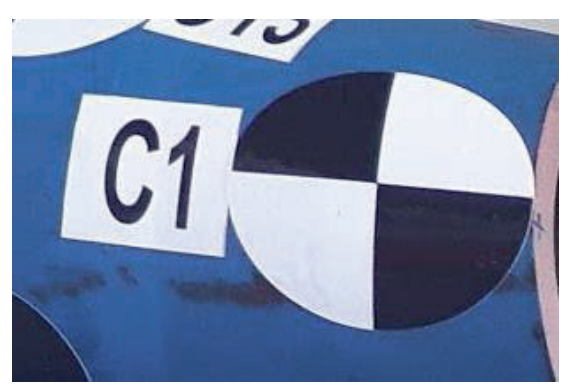

Fig. 2. Example of target used in store separation tests.

At this stage, the scene is still sketched; formal request for support to teams; the definition of the number of test points and their characteristics (some movement determined in the store); 
equipments and test site are reserved. Finally, the campaign is formalized - a document with details of the campaign.

\section{Preparation}

At this stage, all teams and equipment must be positioned at the test site. The store must be positioned on the aircraft pylon. The tires to cushion the impact of the fall are also positioned. The adhesives are placed on the store, pylon and aircraft. At the IPEV, 20 or more targets are glued to a predetermined pattern on the store, pylon and aircraft to allow more precise photogrammetric analysis of the position of the stores.

The cameras are installed in the photographic POD, positioned, configured and their positions are determined (Figure 3).

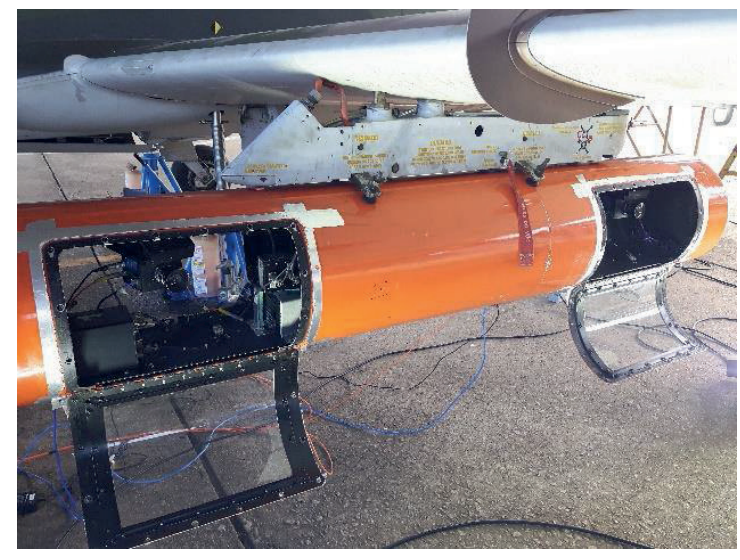

Fig. 3. Cameras installed and positioned inside the $P O D$.

The position of the reflectors, the position and the function of each person at the test site are also defined. The synchronism of the cameras is tested. Some releases are made in order to test the ejector, the trigger, the terminology to be used in the test, the capture of the separation by the cameras. Then, the sketch is validated from the scenario.

\section{Geometry Determination}

The lateral leveling of the aircraft was done with a Tokyo Theodolite TM20C [9]. The longitudinal leveling (Figure 9) was measured by a Nikon Total Station NPL-632 [10], which guided the height adjustment operation of the hydraulic jacks on which the aircraft was supported. The aircraft leveling reference marks were used for this procedure, which was performed as instructed in the Aircraft Maintenance Manual.

For the survey of the coordinates of the points of interest described, it was necessary to establish a topographical polygon close to the aircraft to base the irradiations of the measurements.
With this information, it was possible to measure the geometry of the store, determine the positioning and attitude of the cameras and the geometry of the aircraft.

A key component in this process is the highspeed digital camera. There are many framerates to choose from [1]. However, 200 frames per second is recommended as the best for store separation analysis. A typical charge will travel from its initial captive position to the bottom of the camera view in 0.2 to 0.4 seconds (depending on the distance of the camera and the lens chosen). At 200 frames per second, this will produce 40 to 80 usable frames of data.

In the tests, one Xavante (number 4467) aircraft and two Mikrotron Cube7 high speed cameras were used [11]. The cameras were configured with the acquisition rate at 400 frames per second (fps), mounted externally on the aircraft in a photographic POD to record movements during the release of the store. Two identical cameras were used. This model of camera has the synchronization feature, fundamental in this type of test. This feature allows the cameras to take photos at the same time. Cameras are oriented to maximize overlapping field of view. Because some stores are less than $1.5 \mathrm{~m}$ from the camera and measurement volumes are too large, $6 \mathrm{~mm}$ and $10 \mathrm{~mm}$ lenses are normally used. As a result, optical distortion must be calculated and corrected. For the tests carried out at IPEV, the Kowa $6 \mathrm{~mm}$ C-Mount lens was used [12]. For the tests, an external fuel tank (store) was also used.

\section{Camera Calibration}

There are several approaches for the correction of projective distortion, such as direct linear transformation, polynomial affine transformation, photogrammetric transformation, among others.

As analyzed by [13], the photogrammetric approach is the one that presents the least error, because it uses the camera projection model itself, that is, it considers the principle of the collinearity equations. The problem is that the roll, pitch and yaw $(\varphi, \theta, \psi)$ attitude angles provided by the aircraft system follow the aeronautical definition and are completely different from those used in photogrammetric models. In this sense it is necessary to adapt the reference systems.

The collinearity equations are the mathematical model on which spatial resection is based. They relate the three-dimensional coordinates of the object-space to the corresponding twodimensional space-image, considering the external orientation of the camera. Does not consider the internal orientation, since it is 
defined in the camera calibration process. To do this relationship, these equations consider the location of the camera and its pointing angles, as well as the focal length, not considering the internal geometric distortions of the camera, as shown in Figure 4. In order to arrive at the collinearity equations, besides the change of reference with translations and rotations, a homographic transformation with scale factor should be considered.

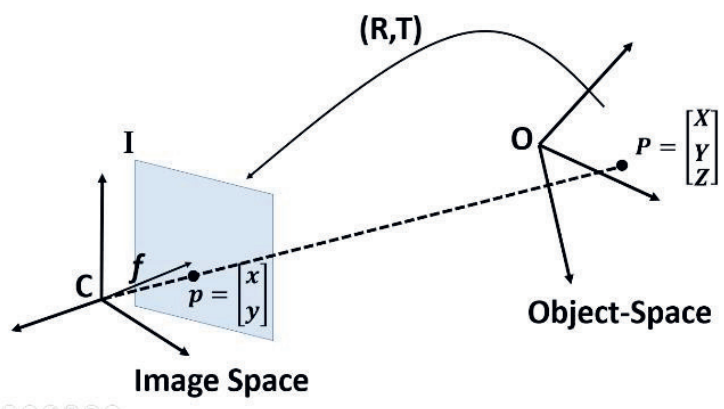

Fig. 4 Change of reference from the system of the object to that of the image.

Where:

C - camera reference system;

I - image reference system;

$\mathrm{O}$ - object reference system;

$f$ - focal length;

$\mathrm{R}$ - rotation matrix;

$\mathrm{T}$ - translation matrix;

$P$ - point in the space of the object;

$X, Y, Z$ - coordinates of point $P$;

$p$ - point corresponding to $P$ in the image space.

$x, y-$ coordinates of the point $p$ in the image system.

All the points that make up the object can be represented in the two-dimensional image space by the principle of collinearity. For objects sufficiently distant from the camera, the image forms in the focal plane, i.e., the points assume coordinate $Z$ equal to the focal distance $f$ in the camera system. Thus, for a camera aligned to the aircraft, the representation of the coordinates in the image system derives from Equation 1 and is given by Equation 2. This happens when the transformation is made by the aeronautical system, Figure 5a. When done by the photogrammetric system (Figure 5b), use equation 3 . The parameters $\lambda_{a}$ and $\lambda_{m}$ are proportionality factors.

$$
\begin{gathered}
{\left[\begin{array}{l}
x \\
y \\
z
\end{array}\right]=R\left[\begin{array}{c}
E-E c \\
N-N c \\
U-U c
\end{array}\right](1)} \\
\lambda_{a}\left[\begin{array}{l}
x_{a} \\
y_{a} \\
f
\end{array}\right]=A\left[\begin{array}{l}
E-E c \\
N-N c \\
U-U c
\end{array}\right]
\end{gathered}
$$

$$
\lambda_{m}\left[\begin{array}{l}
x_{m} \\
y_{m} \\
-f
\end{array}\right]=M\left[\begin{array}{l}
E-E c \\
N-N c \\
U-U c
\end{array}\right]
$$

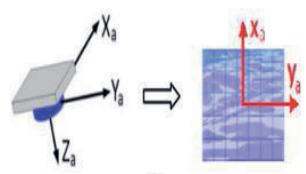

(a)

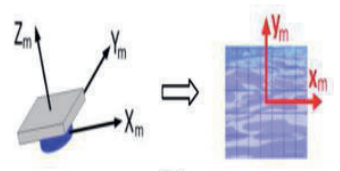

(b)
Fig. 5 (a) Image system when using the aeronautical system (b) when using the photogrammetric system.

In the photogrammetric process, the main task is to establish a strict geometric relation between the image and the object, so that information can be extracted from the object only through the image [14]. However, a raw image contains geometric distortions due to the influence of several intrinsic and extrinsic factors on the sensor. Thus, to obtain reliable metric information from images in the various applications, it is essential that the optical assembly (camera-lenses) be calibrated [15].

\section{Calibration}

Calibration consists of a process for experimental determination of a series of parameters that describe the process of image formation in the camera, according to an analytical model, which relates the known coordinates of a reference grid, also called the calibration field, with the corresponding in the image [16]. There are several field geometries and several methodologies for calibration [17].

Several factors affect the geometric distortion of the images recorded by a sensor, to a greater or lesser degree depending on the architecture of the sensor and on which platform it is inserted. There are geometric aberrations that affect the quality of the image, but not the position of the objects in the image, namely: aberration of sphericity, astigmatism and curvature of the field [18].

The factors are subdivided into internal and external. The first ones are related to the sensor architecture and their distortions are corrected in the process of internal orientation of the image, known as intrinsic parameters. These parameters are determined in the calibration process. The external factors are related to the medium in which the sensor is immersed and its position and pointing in relation to the object of interest, and corrections are corrected in the process of external orientation of the image, known as extrinsic parameters [19]. 


\section{Full Calibration Model}

A model for camera calibration was developed that considers the pinhole model and the radial [20] and tangential distortions [21], which occur when light rays cross the lens before reaching the sensor. The distortion parameters act on the coordinates $(u, v)$ that have no influence on the intrinsic parameters of the matrix $\mathrm{K}$, defined by equation 4.

$K=\left[\begin{array}{ccc}a_{x} & s & \left(c_{x}-c_{c}\right) \\ 0 & a_{y} & -\left(c_{y}-c_{l}\right) \\ 0 & 0 & 1\end{array}\right]$ (4)

Where:

- $c_{c}=(C+1) / 2(5)$

- $l_{c}=(L+1) / 2(6)$

- $K$ is the intrinsic matrix.

- $s$ is the skew parameter.

- $a x$ and ay the affinity terms.

- $\quad c_{x}$ and $c_{y}$ are the principal point lags, in pixels;

- $c_{c}$ and $I_{c}$ are the column and row of the central pixel.

- $\quad C$ and $L$ are the number of columns and rows in the image.

Thus, the model construction begins with the isolated representation of the effects of the radial and tangent distortions, as shown in Equation 7. For the understanding, it is necessary to present Figure 6, an image representation, where the definition of the reference systems and some camera parameters.

$$
\lambda\left[\begin{array}{l}
u \\
v \\
1
\end{array}\right]=M\left[\begin{array}{l}
X \\
Y \\
Z \\
1
\end{array}\right](7)
$$

Where:

$$
\begin{aligned}
& M=\left[\begin{array}{rr}
R & T
\end{array}\right](8) \\
& T=-R\left[\begin{array}{l}
X c \\
Y c \\
Z c
\end{array}\right](9)
\end{aligned}
$$

- $X, Y, Z$ are the axes of the reference system of the calibration field.

- $\mathrm{X}_{\mathrm{c}}, \mathrm{Y}_{\mathrm{c}}, \mathrm{Z}_{\mathrm{c}}$ are the coordinates of the camera CP in the field reference system.

- $\lambda$ is the homographic scale factor.

- $M$ is the reference change matrix.

- $\mathrm{R}$ is the rotation matrix.

- $T$ is the translation matrix.

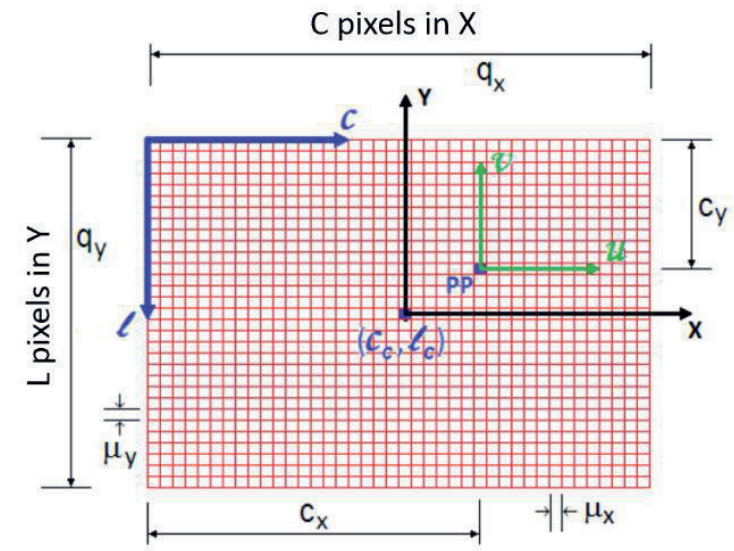

Fig. 6 Reference systems and parameters in the image representation of a camera.

Where:

- $x$ and $y$ are the coordinates in the image system with origin in the central pixel, in pixels.

- $\mathrm{C}_{\mathrm{c}}$ and $\mathrm{I}_{\mathrm{c}}$ are the column and row of the central pixel.

- $\mathrm{u}$ and $\mathrm{v}$ are the coordinates in the image system originating from the main point, in pixels.

- PP is the principal point of collimation, point of intersection of the optical axis with the sensor.

- $\mathrm{c}$ and I are the axes of the column-row reference system of the image, originating from the upper left pixel.

- $c_{x}$ and $c_{y}$ are the lags of the main point, in pixels, measured in the $x$ and $y$ directions in relation to the upper left pixel.

- $\mu_{x}$ and $\mu_{y}$ are the pixel dimensions in the $x$ and $y$ directions.

- $\mathrm{q}_{\mathrm{x}}$ and $\mathrm{q}_{\mathrm{y}}$ are the dimensions of the sensor in the directions $x$ and $y$.

- $\quad C$ and $L$ are the number of columns and rows in the image.

For the geometric correction of the images it was necessary to know some parameters of the camera, some extracted from the manual and others calculated. Figure 7 shows the definition of each of them and the following equations express how they were obtained. 


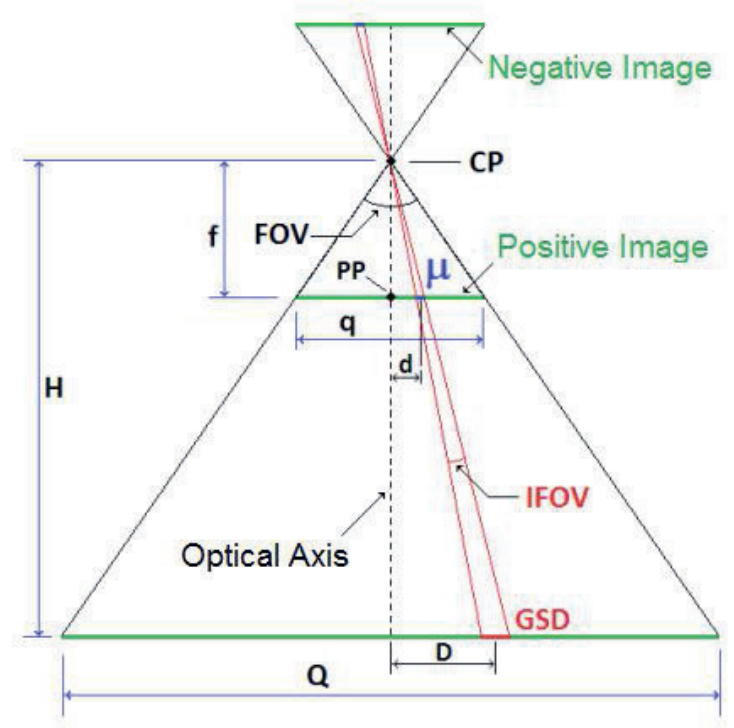

Fig. 7 Geometric parameters of the pinhole camera model.

- $f$ - focal length.

- PP - main point of collimation, intercept of the optical axis with the plane of the image.

- $\mathrm{CP}$ - perspective center, point where the light beams that leave the ground and reach the image.

- $\mu$ - sensor pixel size.

- $\mathrm{q}$ - size of the sensor frame.

- $\mathrm{Q}$ - frame size of the projected image on the ground.

- GSD - Ground Sample Distance - pixel size projected on the ground.

- $\mathrm{D}$ - size of the object on the ground.

- $\mathrm{d}$ - dimension in the image of the object with real dimension $\mathrm{D}$.

- $\mathrm{H}$ - projected distance from the CP to the ground, corresponding to the flight height.

- FOV - Field of View - total aperture angle representing the field of view covered by the sensor.

- IFOV - Instantaneous Field of View - aperture angle representing the field of view of a pixel.

From the geometry of the figure can be extracted the mathematical relations to obtain the geometric parameters. Refer to simplified model of pinhole camera free of internal distortion. In order to obtain the coordinates of the pixels in the system $X_{a}, Y_{a}$, or in the $X_{m}, Y_{m}$, from the coordinates in the row-column system, the affine transformation given by Equations 10 and 11, respectively, is used.

$\left[\begin{array}{l}x_{a} \\ y_{a} \\ f\end{array}\right]=\mu\left[\begin{array}{ccc}-1 & 0 & l_{c} \\ 0 & 1 & -c_{c} \\ 0 & 0 & \frac{f}{\mu}\end{array}\right]\left[\begin{array}{l}l \\ c \\ 1\end{array}\right]$ $\left[\begin{array}{l}x_{m} \\ y_{m} \\ -f\end{array}\right]=\mu\left[\begin{array}{ccc}0 & 1 & -c_{c} \\ -1 & 0 & l_{c} \\ 0 & 0 & -\frac{f}{\mu}\end{array}\right]\left[\begin{array}{l}l \\ c \\ 1\end{array}\right](11)$

\section{Calibration Field}

As seen, the calibration has the objective of identifying the intrinsic parameters of geometric distortion of the cameras, in order to correct the position of the pixels of the points extracted from the images for the spatial resection.

There are several calibration methods that essentially differ in the type and geometry of the field, the number of necessary photographs, the camera positioning method in the field, the quantity and arrangement of distortion parameters in the mathematical model, the adjustment methodology of the model to identify the parameters, among others. Some studies deal with comparative studies of the different calibration methods [17].

Calibrations that require higher accuracy are usually done in laboratory or controlled environments, where target coordinates are precisely known [22]. In this paper, we used an algorithm, which uses this approach [23].

For this, captures are required in a location with known coordinate references. At the IPEV a geometric calibration field was set up in a room in the X-30 hangar, consisting of a threedimensional space with 134 targets, according to Figure 18. The targets are cross-shaped in black and white (soil), blue and white (right wall), red and white (left wall) and green and white (ceiling) (Figure 8). The colors are for easy identification and surveying. The targets were constructed with pieces of $5 \mathrm{~cm}$ of aluminum angle and adhesive with vinyl, each containing the crosshead and its code.

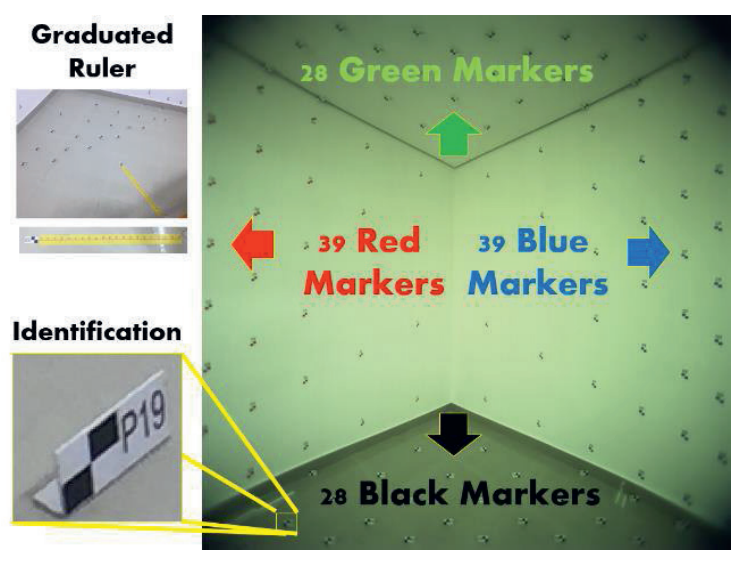

Fig. 8 IPEV Geometric Calibration Field. Detail for identified reference targets and positions marked on the floor for camera positioning. 
It was planned to lay out each target in layers of various depths in order to break the linear dependence that occurs between some parameters of the distortion model.

In the calibration process, the exact 3D coordinates of the targets in the field must be known. Thus, a Total Topographic Station [10] was used to determine the three-dimensional Cartesian coordinates of the 134 targets.

\section{Calibration of Cameras}

The methodology developed for the calibration in this field provides the knowledge of the external orientation of the camera, that is, its position in relation to the targets and their pointing angles. This favors the convergence in the adjustment of the distortion model and the breakdown of linear dependence between some parameters. Photographic shots were taken with the cameras used in pit drop testing. Later, in each image the line-column coordinates of the reference targets were captured, associating the corresponding coordinates in the field with Total Station, and generating an image indicating the location of the captured targets (Figure 9).

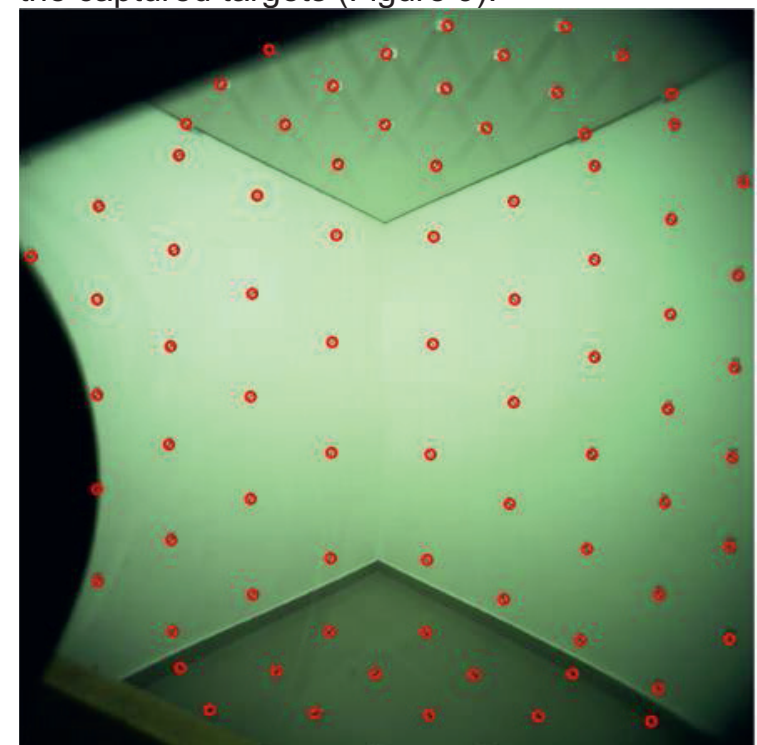

Fig. 9 Examples of captures performed in the IPEV calibration field with the $P O D$ rear camera used in the pitch drop testing.

With the three-dimensional Cartesian coordinates $X Y Z$ of the calibration field targets and the corresponding line-column coordinates in the captured image, the calibration is performed. The radial distortion was adjusted by a polynomial model of six coefficients, according to Figure 10, obtaining a good adhesion.

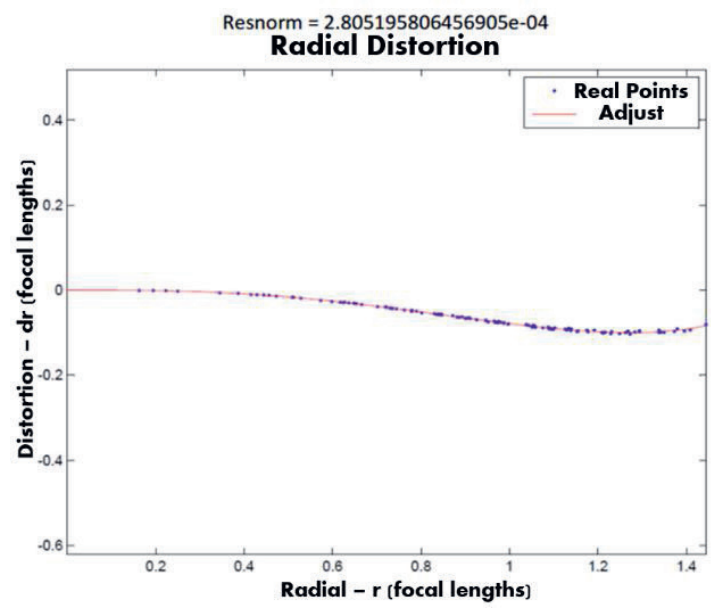

Fig. 10 Radial distortion curve raised in the IPEV field, considering the $P O D$ rear camera.

From the parameters identified in the complete distortion model, the reprojection of the reference targets of the calibration field in the two-dimensional image system was performed, compared to the real coordinates obtained in the capture. This allowed for a qualitative evaluation of the adherence of the model, according to Figure 11

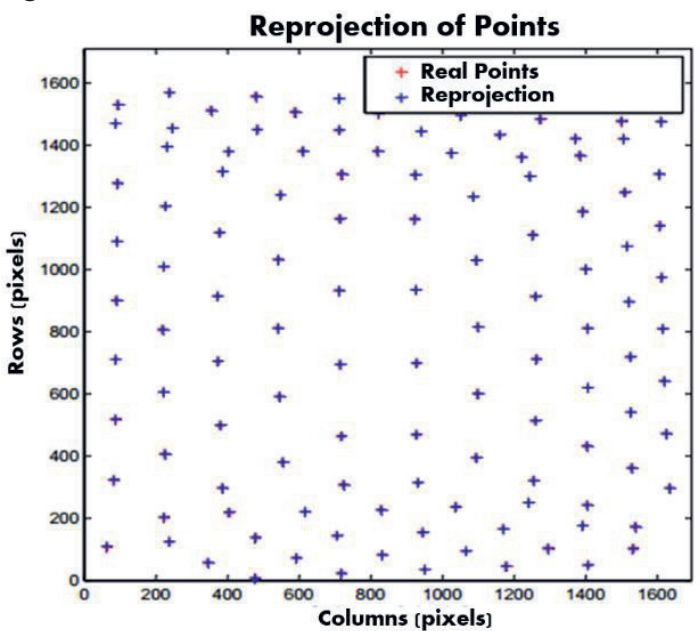

Fig. 11 Reprojection of the reference targets of the calibration field according to the model with the parameters identified for the $P O D$ rear camera in the IPEV field.

With the parameters identified in the calibration process, the images generated by the camera, containing geometric distortions, could be redisplayed pixel by pixel to represent the image that would be generated by a pinhole camera, free of distortions.

The method used was the interpolation by the nearest neighbor, maintaining the original size of the pixel.

\section{Realization of Test Points}

For testing purposes, all equipment must be in place (i.e. tires, store placed on the pylon, 
mounted and attached computers, cameras mounted, connected, configured and synchronized). For each camera there is a computer (notebook) configured. These computers are COTS (Commercial off-theshelf). As soon as the store is ready for release, the cameras are triggered for recording. After this, a trigger is triggered by the test engineer to determine the start of the test point (frame identification on the cameras). When triggered, after 0.5 second the store is released. After the contact of the store on the tires, the cameras are paused. The videos are then downloaded from the cameras and viewed to determine the validity of the videos. If one of the videos was inappropriate (unsynchronized, lost frames, capture crashes, or any other weather), the videos were discarded. Otherwise, the videos are renamed and stored.

After that, go to the next test point, replacing the store on the pylon.

At the IPEV, 10 valid test points were carried out within 2 hours. At the end of the test day, videos are stored on the IPEV data server.

\section{D Analysis}

After performing the test points on the day, the IPEV photogrammetry team works on information processing.

Video files are transferred to a local computer directory for processing. The computer used for processing is a notebook (COTS).

Each video has, in general, 0.5 second of images that precede the beginning of the separation and 0,5 second of images after the contact of the store in the tires. These images are unnecessary in rendering. Thus, another algorithm was developed to determine automatic recognition for discarding unnecessary images.

The next step is to define the center of each target (store, pylon and wing) so that they can be tracked during separation. To do this, the region of interest is delimited with the aircraft's store and pylon. This is possible because the position of the store and the pylon relative to the camera will not be changed considerably between the separations. The positions of each target are previously known and measured from a total station [10]. An identification algorithm scans the image, looking for corners. It can be observed in Figure 12 that 19 targets were found (red dots) in the store, 7 targets in the pylon and 13 targets in the wing, considering an camera on the left inside the POD.

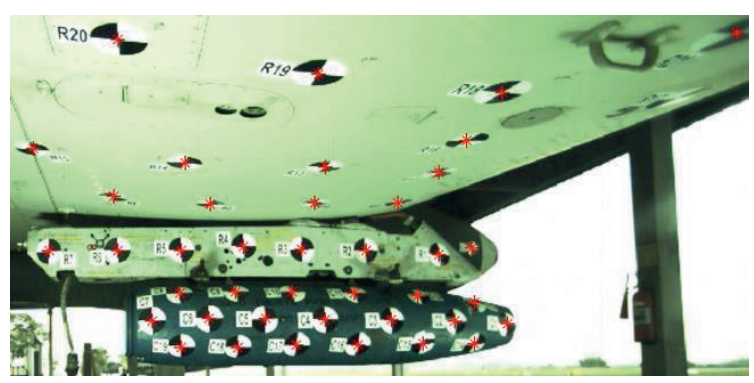

Fig. 12 Targets identified in store, pylon and wing.

In Figure 13 it is possible to observe the aircraft, pylon and store targets that were tracked during the test point.

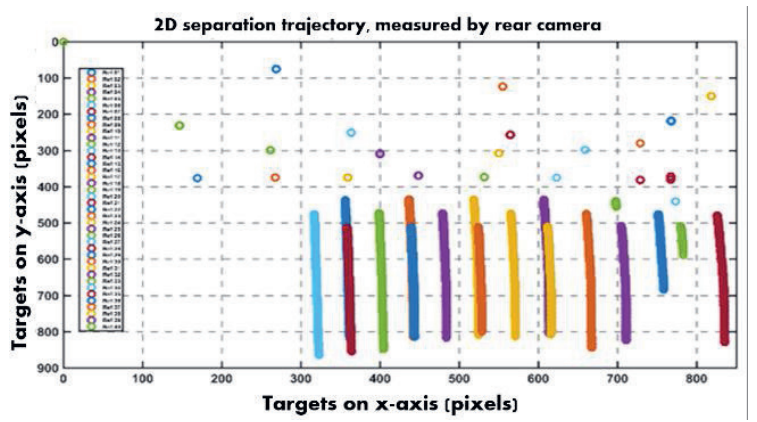

Fig. $132 D$ separation trajectory measured by the rear camera.

Given the 2-D frames of each camera, the problem of solving the position $(x, y, z)$ of each target is solved using least squares. Each target is defined by the intersection of two lines, generated by the line of sight of each camera. Each line in space is represented by 2 equations in $x, y$ and $z$, so that with two straight lines (4 equations), we have an over-determined system, which is solved by least squares (4 equations and 3 unknowns). With a third camera, we have two more equations. It could quickly determine, by means of determinant, if there is intersection between 2 lines in space, which will hardly occur in the experimental measurements. However, a middle ground can be obtained by resolution in the least squares (a point that does not belong to any line but is closest to both simultaneously).

\section{D Analysis}

For the multicamera triangulation algorithm, two or more cameras are used to quantify the error. In the case of POD used, only 2 cameras are possible. After executing the algorithm of $2 \mathrm{D}$ Analysis, it is necessary to perform 3D Analysis.

Suppose we are given the coordinates of a number of points measured in two different Cartesian coordinate systems (Fig. 14) [24]. The photogrammetric problem of recovering the transformation between the two systems from these measurements is called absolute orientation [25]. This problem occurs in various contexts. 
The transformation between two Cartesian coordinate systems can be thought of as the result of a rigid body movement and can thus be decomposed into a rotation and a translation. In stereophotogrammetry, moreover, the scale may not be known. There are obviously three degrees of freedom for translation. The rotation has another three (direction of the axis on which the rotation occurs plus the angle of rotation around this axis). Scaling adds one more degree of freedom. Three known points in both coordinate systems provide nine constraints (three coordinates each), more than enough to allow the determination of the seven unknowns ( 3 translations, 3 rotations and 1 scale).

Discarding two of the constraints, seven equations in seven unknowns can be developed to allow the retrieval of parameters.

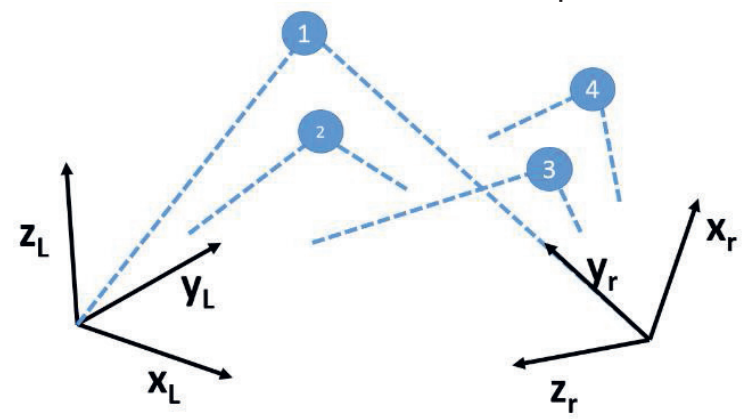

Fig. 14 The coordinates of a number of points is measured in two different coordinate systems. The transformation between the two systems is to be found. Adapted from [24].

The algorithm implemented by [24], uses all available information to obtain the best possible estimate (in a least squares sense). In addition, it is preferable to use it for center point estimation, rather than rely on single-point or single-point measurements. This algorithm is used in this work.

The main purpose of $3 \mathrm{D}$ analysis is to obtain $6 \mathrm{DoF}$ data. In Figure 15 is shown the example of the view of a test point considering $X, Y, Z$.

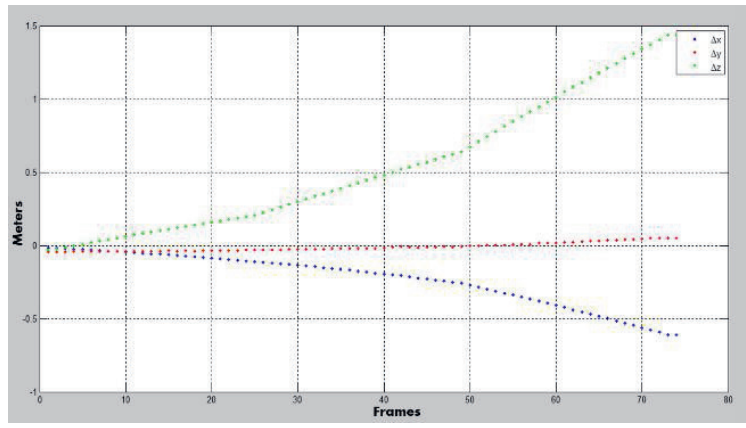

Fig. $15 X, Y, Z$ data from a test point.

Figure 16 shows the angles $(\varphi, \theta, \psi)$.

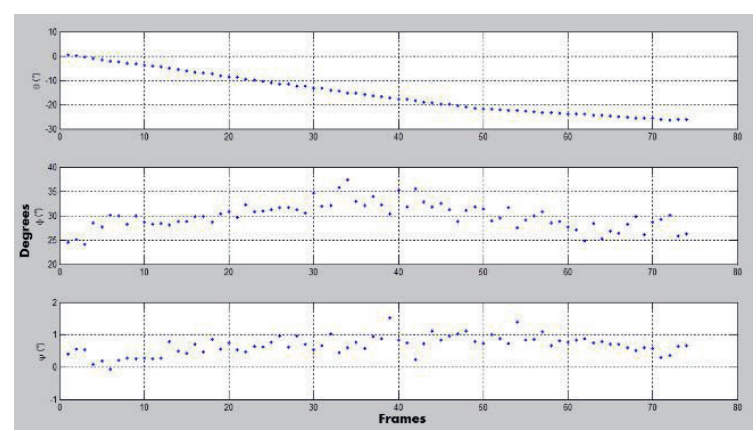

Fig. 16 Data in roll, pitch, yaw of a test point..

\section{Considerations}

The objective of this paper was to show the implementation of the method and the photogrammetric solution, developed by IPEV, to perform the analysis of pitch drop tests.

The proposed solution is promising. Both method and solution obtained errors of the order of $\mathrm{mm}$ and were considered acceptable for this type of test.

This solution is part of an IPEV development program that aims to perform the real-time analysis in store separation flight tests using photogrammetry.

Suggestions for future work are:

- Validate the information obtained by the photogrammetric solution with another solution widely known in the market (e.g. TrackEye).

- Perform new load separation tests on soil.

- Continue the development of the in-flight test program with real-time analysis.

\section{References}

[1] Arnold, R.J. and Epstein, C.S., AGARD Flight Test Techniques Series on Store Separation Flight Testing. Volume 5. NATO. No.300. Published April 1986. ISBN 92-835-1523-4.

[2] Persson, I. and Lindberg, A., Transonic Store Separation Studies on the SAAB Gripen Aircraft Using Computational Aerodynamics. SAAB Aerosystems. 26th International Congress of the Aeronautical Sciences. ICAS 2008.

[3] Cenko, A., Store Separation Lessons Learnded During the Last 30 Years, NAVAIR, Patuxent River, MD 20670. 27th International Congress of the Aeronautical Sciences. ICAS 2010.

[4] Carman, J. B., Hill, D. W., Christopher, J. P., Store Separation Testing Techniques at the Arnold Engineering Development Center. Volume II: Description of Captive Trajectory Store Separation Testing in the Aerodynamic Wind Tunnel (4T). AEDC-TR-79-1, Vol. II (ADA087561). 1980.D 
[5] Cho, H., Kang, C., Jang, Y., Lee, S. and Kim, K., Store Separation Analysis of a Fighter Aircraft's External Fuel Tank. Int'I J. of Aeronautical \& Space Sci. 11(4), 345-350 (2010). doi:10.5139/IJASS.2010.11.4.345

[6] MIL-HDBK-1763, Aircraft/Stores Compatibility: Systems Engineering Data Requirements And Test Procedures. Department of Defense USA. 1998.

[7] Getson, E.S., Telemetry Solutions for Weapon Separation Testing. 33th Annual International Symposium. 2002.

[8] North Atlantic Treaty Organization, Aircraft/Stores Compatibility, Integration and Separation Testing, STO AGARDograph 300, Flight Test Technique Series - Volume 29. AG-300-V29. Published September 2014.

[9] LIETZ, Double Center Theodolite TM-20C, 2018. Available in https://cn.sokkia.com/sites/default/ files/sc_files/downloads/tm20c_0.pdf. THE LIETZ COMPANY.

[10] NIKON, Total Station DTM-322 - Instruction Manual, Available in http://www.mcesurvey.com/files/Nikon_DTM322_Total_Station_Manual.pdf. Version A 1.0.0, Part Number C232E, April 2009.

[11] MIKROTRON, Mikrotron Cube7, Available in https://mikrotron.de/fileadmin/Data_Sheets/HighSpeed_Recording_Cameras/mikrotron_motionblit z_eosens_cube7_datasheet.pdf. 2018.

[12] KOWA, Lenses $6 \mathrm{~mm}$ Kowa, Available in http://www.rmaelectronics.com/content/KowaLenses/LM6HC.pdf. 2018.

[13] Lima, S. A.; Brito, J. L. N. S., Estratégias para retificação de imagens digitais. Em: COBRAC 2006 - Congresso Brasileiro de Cadastro Técnico Multifinalitário, UFSC, Florianopolis, 2006. Anais, Artigos, n. 90, p. 1-14, 2006.

[14] Mikhail, E. M., Bethel, J.S. and McGlone, J.C., Introduction to Modern Photogrammetry. Hoboken: Wiley, 2001. 479p. ISBN 978-81-2653998-7.

[15] Gruen, A.; Huang, T. S, Calibration and orientation of cameras in computer vision. Berlin: Springer, 2001. 235 p. ISBN (3-540-65283-3).

[16] Swapna, P.; Krouglicof, N.; Gosine, R., The Question of Accuracy with Geometric Camera Calibration, St. John's, Newfoundland, pp. 541546, May 3-6, 2009.

[17] Clarke, T.A.; Fryer, J.F., The development of camera calibration methods and models, Photogrammetric Record, 1998, 16(91): pp 51-66.

[18] Brito, J.L.N.S.; Coelho, L.C.T.F., Fotogrametria Digital, Rio de Janeiro: Ed UERJ, 2007. 196p. ISBN 978-85-7511-114-7.

[19] Schenk, T., Digital Photogrammetry, Ohio, USA: TerraScience, $1^{\text {a }}$ ed, 1999.
[20] Heikkila, J., Camera Calibration Toolbox for Matlab. $2000 . \quad$ Available in http://www.vision.caltech.edu/bouguetj/calib_doc/

[21] Brown, D.C., Decentering Distortion and the Definitive Calibration of Metric Cameras, 29 ed, Washington DC, pp 444-462, March 1965.

[22] Ladstadter, R.; Gruber, M., Geometric aspects concerning the photogrammetric workflow of the digital aerial camera UltraCamX. In: International Congress for Photogrammetry and Remote Sensing, 21., 2008, Beijing, China. Proceedings... Beijing: International Society of Photogrammetry and Remote Sensing (ISPRS), 2008. p. 521- 526. Vol XXXVII part B1. ISSN (1682-1750).

[23] Roberto, L., Acurácia do p osicionamento e da orientação espacial de veículos aéreos a partir de imagens de câmeras de $\mathrm{p}$ equeno formato embarcadas. - São José dos Campos : INPE, 2017. 308p. Dissertação (Mestrado em Sensoriamento Remoto) - Instituto Nacional de Pesquisas Espaciais, São José dos Campos, 2017.

[24] Horn, B. K. P., Closed-form solution of absolute orientation using unit quaternions, Vol. 4, No. 4/April 1987/J. Opt. Soc. Am. A

[25] Slama, C. C. Slama, Theurer, C., and Henrikson, S.W., Manual of Photogrammetry (American Society of Photogrammetry, Falls Church, Va., 1980). 